\title{
A Systematic Review Study on the Changer Factors of the Quality of life in Cancer Patients
}

\author{
Mostafa Madmoli ${ }^{1 *}$
}

Emergency Medical Technician, Dezful University of Medical Sciences, Dezful, Iran*

\begin{abstract}
Introduction: The rate of mortality from cancer is increasing and at least about 8 million people die every year because of cancer. Unlimited proliferation potential, reduced apoptosis, increased angiogenesis, tissue invasion and metastasis are factors of cancer progression. The purpose of this study was a systematic review study on the changer factors of the quality of life in cancer patients.

Materials and Methods: This is a systematic review which in order to achieve the study goal and to improve the accuracy of the study and a comprehensive understanding of this, this integrated overview study was conducted based on the Broome method. The method is done in the form of three steps in the search of texts, data evaluation and data analysis, So, in the search phase, post-retrospective study texts are examined in four stages in terms of inclusion criteria. After obtaining the conditions for entry into the study, the content of the study is evaluated and at the end was done the analysis of the data.

Results: In this study, 20 papers were reviewed that The factors that change the quality of life of patients with cancer are shown. The results of one of these studies showed that the hope-centered therapy group, has increased the mean scores of functional domain and quality of life in patients in the intervention group. However, there was no significant difference in symptoms.

Conclusion: According to the studied studies, we can say whose therapeutic hope has a significant impact on improving the various dimensions of health-related quality of life in men and women with cancer. Thus, the authorities and managers of the health system of the country, using the results of research in this field, by implementing programs and strategies to create hope-based group therapy interventions for cancer patients, they can improve their quality of life in different areas. The educational pamphlet on occupational therapy programs also affected many of the quality of life of women with breast cancer that it is better to take the necessary measures in this case too.
\end{abstract}

Keywords: changer factors, Quality of Life, Patients with Cancer

\section{Introduction}

Cancer is currently a major public health problem in the world. The incidence of cancer deaths is increasing and at least about 8 million people die every year because of cancer. Unlimited proliferation potential, reduced apoptosis, increased angiogenesis, tissue invasion and metastasis are factors of cancer progression. One of the causes of cancer is diabetes. Diabetes is a chronic metabolic disease that accounts for 15 percent of the health care costs in the United States. Increasing blood glucose causes early and

Address For Correspondence:

Mostafa Madmoli, Emergency Medical Technician, Dezful University of Medical Sciences, Dezful, Iran. E-mail: mostafamadmoli10@yahoo.com

Received: 02-01-2019 Revised: 24-02-2019_Accepted: 04-03-2019 
late complications of diabetes (1-7). Complications of diabetes are very common among patients. Foot complications in people with

Diabetes characterized by chronic hyperglycemia that damage affects the individual's individual members and reduces longevity. Complications of diabetes, while causing high costs for individuals and society, it also leads to an increase in mortality in people with diabetes. Lifestyle behaviors, including nutritional behaviors, play an important role in preventing and managing diabetes (15-21).

One of the disorders found in diabetic patients is depression and occupational stress. Which is one of the most common psychiatric disorders in diabetic patients that in addition to the effect on the course of treatment, it can change the prognosis of the disease $(22,23)$. Job stresses, physical and emotional responses are harmful that when the requirements of the work do not correspond to the capabilities, resources or needs of the worker, they occur. This may lead to illness and injury and is known as a known psychosocial agent in the development of cardiovascular disease $(24,25)$.

Diabetes also affects thalassemic patients. Endocrine disorders such as diabetes mellitus have a significant prevalence in thalassemic patients. Many studies have shown this that the cause of these disorders can be the accumulation of iron in the tissues of the body, such as pancreas (26-30).

In general, diabetes can have multiple complications and cause various diseases. The purpose of this study was a systematic review study on the changer factors of the quality of life in cancer patients.

\section{Materials and methods}

This study is a systematic review that using articles published in the last 27 years about diabetes have various muscular and neuropathic musculoskeletal disorders and circulatory disorders (8-14).

the factors that altered the quality of life of cancer patients. It was conducted in English and Persian by searching articles in search engines, authoritative scientific sites and databases SID, Google Scholar, Embase, ResearchGate, Sciencedirect, PubMed, Springer.In the first stage, 44 articles were found. Of these, 20 articles related to the topic that were published in the last 27 years were reviewed.

In order to achieve the study goal and to improve the accuracy of the study and a comprehensive understanding of this, this integrated overview study was conducted based on the Broome method. The method is done in the form of three steps in the search of texts, data evaluation and data analysis, So, in the search phase, post-retrospective study texts are examined in four stages in terms of inclusion criteria. After obtaining the conditions for entry into the study, the content of the study is evaluated and at the end was done the analysis of the data.

The studies studied were in English or Persian, access to their full text was possible and they were published over the last 27 years, Entered the study and unnamed studies were deleted without history and nonacademic.

To achieve relevant studies, a wide range of keywords including changer factors, Quality of Life, Patients with Cancer was used as a one-to-one search, combined with the method "And" and "OR".

\section{Results}

Cancer is currently a major public health problem in the world. The incidence of cancer deaths is increasing And at least about 8 million people die every year because of cancer (4-7). In this study we intend to review 20 articles, understand The factors that change the quality of life in cancer patients. 
In a study that was done, shows the use of reflexology in patients with breast cancer can improve quality of life. Comparison of mean score of life quality before and two weeks after intervention in both the experimental and placebo groups showed a significant difference. The mean of total quality of life score in the two weeks after the intervention was significantly higher in the test group than in the placebo group. The results also showed that there was a significant difference in overall quality of life scores between the three groups of test and placebo and control after intervention (31).

In another study, which was done, group therapy has reduced anxiety and depression and increased quality of life in cancer patients. In this study, the findings indicated a significant decrease in mean scores of anxiety and depression and a significant increase in the mean scores of quality of life in the experimental group compared to the control group (32).

In a study that was done, showed that after the independent variable (Spirituality Therapy), the quality of life scores of women with breast cancer in the experimental group was higher and the difference was significant with the control group and It also shows the results of the analysis of the quality of life subscale in three sub-scales (area of physical health, mental health and social relation), the mean score of the experimental group in the post-test was more than the control group and was statistically significant (33).

In another study, which was done, there was a significant difference in mean of fatigue and quality of life according to hemoglobin level. There was a significant negative correlation between hemoglobin level and quality of life and severity of fatigue (34).

In a study that was done, significant statistical differences were reported in the sub-scales of quality of life, general health status, physical function, emotional functioning, role function, social function, mental image and futurism between the two groups after intervention.
There is a significant difference in other subscales of cognitive function, sexual function and sexual pleasure, as well as pain symptoms, dyspnea, diarrhea, and financial problems were not reported between the two groups of test and control (35).

In a study that was done, educational pamphlets on occupational therapy programs affected many of the issues related to the quality of life of women with breast cancer (36). In a study that was done, that the results of the data indicate a significant difference between the experimental group and the quality of life scale and sub-general health, emotional performance, sexual performance, and symptom relief (37).

The results of the study, which were done, shows that the hope-centered group therapy has increased the mean scores of functional domain and quality of life among patients in the intervention group. However, there was no significant difference in symptoms (38). The results of the study, which were done, shows That by performing group motivational interviews, there is a significant difference in the quality of life score of patients with intestinal cancer with permanent atrophy (39).

The results of these studies (40-49), shows that spirituality therapy can be an appropriate treatment for improving the quality of life and physical, psychological and social dimensions of patients suffering from breast cancer. Findings of the research (50), shows that the feeling of tiredness was the most obvious symptom of the disease itself or the complications of the disease in those with breast cancer. Having a child had a positive effect on the quality of life of these patients. The feeling of tiredness was the most obvious symptom of the disease itself or the complications of the disease in those with breast cancer.

\section{Discussion}

Cancer is currently a major public health problem in the world. Unlimited proliferation 
potential, reduced apoptosis, increased angiogenesis, tissue invasion and metastasis are factors of cancer progression (4-7). The purpose of this study was a systematic review study on the changer factors of the quality of life in cancer patients.

According to study (31), that showed that the use of reflexology in patients with breast cancer can improve quality of life, breast cancer is one of the most common cancers in women. Assessing the quality of life is an important issue in the study of chronic diseases, especially cancer. The entire set of physical and psychological symptoms of cancer affects the quality of life of patients. Use of complementary therapies including reflexology for patients with pain and emotional discomfort from cancer can lead to a feeling of relaxation in patients.

According to study (32), that showed that group psychometrics reduced anxiety and depression and improved quality of life in patients with cancer, it should be said that spiritual therapy means taking into account the cultural and religious beliefs of people in the process of treatment and taking into account the high level of clients. Leading them to a transcendental source. The importance of spirituality and spiritual growth in humans has been increasingly considered by psychologists and mental health professionals in the last few decades.

According to study (36), that showed that the educational pamphlet of occupational therapy programs affected many of the issues related to the quality of life of women with breast cancer, breast cancer is one of the most common cancers in women. Common therapies are cancer, surgery, radiation therapy, chemotherapy and hormone therapy. Despite the increased chance of living with these treatments, multiple psychiatric, social and physical complications remain. Which often leads to problems in doing activities and tasks such as housekeeping. This study showed that therapeutic pamphlet is effective in these patients and should be used in subsequent studies.
Also, according to the study (39), that Showed that by performing group motivational interviews, there was a significant difference in the quality of life score of patients with intestinal cancer with permanent ostomy, it can be said ostomy Patients are involved with many physical, psychological and social problems. Problems have disturbed the natural process of their quality of life. So that they experience lower quality of life due to their particular circumstances. Therefore, proper training and interventions can improve the quality of life of these patients.

\section{Conclusion}

According to the studied studies, we can say whose therapeutic hope has a significant impact on improving the various dimensions of health-related quality of life in men and women with cancer. Thus, the authorities and managers of the health system of the country, using the results of research in this field, by implementing programs and strategies to create hope-based group therapy interventions for cancer patients, they can improve their quality of life in different areas. The educational pamphlet on occupational therapy programs also affected many of the quality of life of women with breast cancer that it is better to take the necessary measures in this case too.

\section{Acknowledgment}

The author of this article is grateful to all those who have collaborated with me in this article.

\section{References:}

1. Moslemirad M, Madmoli M, Madmoli Y, Niksefat M. Prevalence of type 1 and type 2 diabetes and its related factors in diabetic patients hospitalized in Khatamol-Anbia hospital in Shoushtar, 2014-15: A retrospective study. Journal of Research in Medical and Dental Science. 2018 May 22;6(3):421-6. 
2. Madmoli M, Eilami O, Rezaie K, Aliabad MA, Moslemirad M. Diabetes and the risk of suffering cardiovascular Diseases: A two-year retrospective study. International Journal of Ecosystems and Ecology Science (IJEES). 2018 Jun;8(3): 649-56.

3. Madmoli M Rostami F, Mirsami Yazdi N, Mosavi A, Baraz Sh. Evaluation of Prevalence of Diabetic Foot Ulcer and Its Related Factors in Diabetic Patients Admitted to KHatam-ol-Anbia Hospital in Shoushtar During 2015-2016: A Retrospective Study. International Journal of Ecosystems and Ecology Science (IJEES). 2018 June; 8 (3): 545-52.

4. Mostafa Madmoli, Mehdi Fallah bagher shaidaei, Akram Rohani, Pouriya Darabiyan, Fariba Mobarez. The correlation between alcohol consumption and reducing the age of cancer incidence in patients with this disease. Medical Science, 2019, 23(95), 48-53

5. Rostami F, Madmoli M, Mirsami Yazdi N, Baraz Sh. Evaluation of The Prevalence of Lower Limb Amputation and Its Related Factors in Diabetic Patients Admitted to KHatam-ol-Anbia Hospital in Shoushtar During The 2015-2016: A Retrospective Study. International Journal of Ecosystems and Ecology Science (IJEES). 2018 June; 8 (3): 553-60.

6. Raisifar Z, Afshar Nia A, Madmoli M, Madmoli Y. The Relationship Between Using Insulin and Suffering Alzheimer's Disease in Patients with Diabetes: A TwoYear Study. International Journal of Ecosystems and Ecology Science (IJEES). 2018 June; 8 (3): 623-28.

7. Shirali M, Madmoli Y, Roohafza J, Karimi $\mathrm{H}$, Baboli Bahmaei A, Ertebati S. Improvement diagnosis of diabetes using a combination of sugeno fuzzy inference systems and firefly algorithms. ijdld. 2017; 15 (3): 172-176. [in persian].

8. Raisifar Z, Afshar Nia A, Maghamesi Moarrefi H, Madmoli M. Evaluation of Gi Bleeding Prevalence and Its Related Factors in Diabetic Patients Hospitalized in KHatam-ol-Anbia Hospital During 2015-16: A Retrospective Study. International Journal of Ecosystems and Ecology Science (IJEES). 2018 June; 8 (3): 609-14.

9. Mostafa Madmoli, Zahra Kord, Azita Bandani, Negin Sedighi, Mahla Rezaei Shandiz, Pouriya Darabiyan, Alieh AfsharNia. Epidemiological and clinical study of patients with Alzheimer's in Five Cities of Khuzestan Province in 20162018. Medical Science, 2019; 23(95), 1-5

10. Hadi Mashali, Fatemeh Toleideh, Rezvan Rahmani, Pouriya Darabiyan, Mostafa Madmoli. The predictive role of Hyperlipidemia in the incidence of ACS in patients referring to Shahidzadeh Hospital in Behbahan in 2016 -2017. Medical Science, 2018; 22(94), 566-570

11. Mostafa Madmoli, Yasaman Modheji, Alireza Rafi, Rezvan Feyzi, Pouriya Darabiyan, Alieh AfsharNia. Diabetes and its predictive role in the incidence of Alzheimer's disease. Medical Science, 2019; 23(95), 30-34

12. Madmoli M. Quality of Life in Patients with Cancer and Some Factors Affecting it: A Systematic Review. International Journal of Research Studies in Science, Engineering and Technology. 6(1), 2019; 1-7

13. Madmoli M. Clinical and Laboratory Findingin Children with Leukemia: a Systematic Review. International Journal of Research Studies in Science, Engineering and Technology, vol. 5, no. 10, pp. 1-6, 2018.

14. Madmoli M, Madmoli Y, Khodadadi M, Samsamipour M. Some Factors Affecting Quality of Life in Patients with Diabetes: A systematic Review. Annals of Microbiology and Infectious Diseases. 2019; 2(1). 26-30.

15. Mostafa Madmoli, Zahra Mahmoudi Dehcheshmeh, Alireza Rafi, Zahra Kord, Fariba Mobarez, Pouriya Darabiyan. The rate of some complications and risk factors of diabetes in diabetic patients: 
Study on cases of 3218 diabetic patients. Medical Science, 2019; 23(95), 63-68

16. Mostafa Madmoli. Evaluation of Chemotherapy Complications in Patients with Cancer: A systematic Review. International Journal of Research Studies in Science, Engineering and Technology, vol. 5, no. 12, pp. 59-64, 2018.

17. Mostafa Madmoli, Mehran Yarbig, Negin Sedighi, Pouriya Darabiyan, Fariba Mobarez. Communication between body mass index and the risk of obesity-related cancer: A 5-year study on patients with cancer. Medical Science, 2019; 23(95), 6974.

18. Madmoli M. Quality of Life in Patients with Cancer and Some Factors Affecting it: A Systematic Review. International Journal of Research Studies in Science, Engineering and Technology. 6(1), 2019; 1-7

19. Madmoli M, Madmoli Y, Khodadadi M, Samsamipour M. Study of Some Effective Treatments for Accelerating Diabetic Foot Ulcer Healing: A Systematic Review. International Journal of Research Studies in Science, Engineering and Technology, 6(2), 2019; 34-39

20. Madmoli M, Madmoli Y, Khodadadi M, Samsamipour M. Factors Affecting the Level of Glycosylated Hemoglobin in Patients with Diabetes: A Systematic Review. Annals of Microbiology and Infectious Diseases 2(1), 2019; 43-47.

21. Madmoli M, Madmoli Y, Khodadadi M, Samsamipour M. Some Factors Affecting Quality of Life in Patients with Diabetes: A systematic Review", Annals of Microbiology and Infectious Diseases, 2(1), 2019; 26-30.

22. Madmoli Y, Madmoli M, Qashqaei nezhad $\mathrm{N}$, Bosak S. Prevalence of depression and associated factors in adolescents of masjedsoleyman. JPEN. 2016; 2(4):31-40.

23. Madmoli M, Madmoli Y, Bigdeli Shamloo MB, Etebari A, Mahmoodi Kouhi A, Azami M. The Relationship Between Depression and Religiousness in Female High School Students of Masjed Soleyman in 2015.
Journal of Pediatric Nursing. 2017 Jun 15;3(4):15-22.

24. Roughani A, Madmoli M, Raisifar Z, Kikhavani S, Yasemi M, Azami M, Sharifi Z. The prevalence of behavioral disorders and its related factors in elementary school children in Ilam City in 20112012. Indian Journal of Forensic Medicine \& Toxicology. 2018;12(4):165-9.

25. Madmoli M, Nikpay S. An Investigation of the Relationship between Spiritual Health and Depression, Anxiety, and Stress among Students of ilam University of Medical Sciences. Journal of Research in Medical and Dental Science. 2018 May 17;6(3):294-300.

26. Madmoli Y, Aslani A, Ahmadi Y, Mousavi M, Mashalchi H, Niksefat M, Madmoli M. Study Habits and Related Factors in Students of Nursing and Midwifery College of Dezful University of Medical Sciences in 2015. Iranian Journal of Nursing Research (IJNR). 2017.

27. Madmoli Y, Akhaghi Dezfuli SM, Beiranvand R, Saberi Pour B, Azami M, Madmoli M. An epidemiological and clinical survey of patients with $\beta$ thalassemia in dezful in 2015. Iranian Journal of Epidemiology. 2017;13(2):14552.

28. Maraghi E, Adavi A, Madmoli Y, HeidariSoureshjani R, Madmoli M. The Effect of Orem Self-Care on Mental Health of Patients with Thalassemia Major. Journal of Clinical Nursing and Midwifery. 2018;4(3).

29. Madmoli M, Madmoli Y, Rahmati P, Adavi A, Yousefi N, Gheisari Z, Abbaszade Aliabad M. Quality of Life and Some Related Factors in Patients with Beta Thalassemia Major in Southwest Iran. Journal of Client-centered Nursing Care (JCCNC. 2017;3(2).

30. Madmoli Y, Akhaghi Dezfuli SM, Adavi A, Maraaghi E, Heidari Soureshjani R, Madmoli M. The Effect of Orem Self-Care on Mental Health of Patients with Thalassemia Major. Journal of Clinical 
Nursing and Midwifery. 2018 Jul 1;7(2):108-15.

31. Haghighat $\mathrm{S}$. The effect of Reflexology on Quality of Life of breast cancer patients during chemotherapy Iranian Journal of Breast Diseases.34-23: (1) 6 ;2013

32. Hamid N, Talebian L, Mehrabizadeh Honarmand M, Yavari AH. The Effect of Semanticism on Depression, Anxiety and Quality of Life in Cancer Patients. Journal of Psychological Achievements. Sep 17, 2013; 4 (2).

33. mohamad karimi $M$, shariatnia $K$. EFFECTIVENESS OF SPIRITUAL THERAPY ON THE LIFE QUALITY OF THE WOMEN WITH BREAST CANCER IN TEHRAN. J Urmia Nurs Midwifery Fac. 2017; 15 (2) :107-118

34. Zeighami Mohammadi S, Houshmand $P$, Djafari ${ }^{2}$ F, Esmaily H, Kooshyar M M. The relationship between anemia and severity of fatigue and quality of life in cancer patients undergoing chemotherapy. MEDICAL SCIENCES. 2011; 20 (4) :265272.

35. Afrasiabifar A, Hamzhiekia S, hosseini N. The effect of self-care program using Orem's self- care model on the life quality of women with breast cancer undergoing chemotherapy: a randomized controlled trial. Armaghane danesh. 2018; 23 (1) :113.

36. Ghanbari S. Survey of the effect of Occupational Therapy program pamphlet on Quality of Life in women with Breast Cancer Iranian Journal of Breast Diseases.49-43. (2);2013 .

37. Shoa Kazemi M. The Effectiveness of Four Than Psychotherapy (Awareness, Hope, Relationship Therapy and Behavior Regulation) on Improving the Quality of Life of Women with Breast Cancer. Journal of Breast Disease. 2012; 5 (2 and 3): 50-59.

38. molaei Z, Ahangarzadeh Rezaei S, Khalkhali H R. THE EFFECT OF HOPEBASED GROUP THERAPY ON THE CANCER PATIENTS` QUALITY OF LIFE BASED ON SNYDER'S HOPE THEORY. J
Urmia Nurs Midwifery Fac. 2017; 15 (6) :404-414.

39. Rajabipour E, Maddah S, Falahi Khoshknab M, Zarei F, Anaraki F. Effect of group motivational interviewing on quality of life of patients with colorectal cancer and permanent ostomy. IJPN. 2014; 2 (3) :58-68.

40. Canada AL, Parker PA, de Moor JS, BasenEngquist K, Ramondetta LM, Cohen L. Active coping mediates the association between religion/spirituality and quality of life in ovarian cancer. Gynecol Oncol 2006;101(1):102-7.

41. Livneh H, Erin M, Todd B. Psychosocial Adaptation to Chronic Illness and Disability: A Preliminary Study of its Factorial Structure Journal of Clinical Psychology in Medical Settings 2004;13(3): 250-60.

42. Litwinczuk KM, Groh CJ. The relationship between spirituality, purpose in life, and well-being in HIVpositive persons. J Assoc Nurses AIDS Care 2007;18(3):13-22.

43. Babayi Z, latifi Z. Spirituality effectiveness of therapy on quality of life and mental health in women with diabetes. Ravanshenasi va Din; 2015; (2): 37-50. (Persian).

44. Larson CAD. Spiritual, psychosocial, and physical correlates of well-being across the breast cancer experience. (Dissertation). Tucson, AZ: University of Arizona; 2004.

45. Lim JW, Yi J. The effects of religiosity, spirituality, and social support on quality of life: a comparison between Korean American and Korean breast and gynecologic cancer survivors. Oncol Nurs Forum 2009; 36: 699-708.

46. Rahmati M, Falahi Km, Rahgouy M, Rahgozar M. The effect of spiritualreligious group therapy on mental status of schizophrenic inpatients. Nurs Res 2010;5: 13-20. (Persian).

47. Hojjati H, Motlagh M, Nuri F, Sharifnia H, Mohammadnejad E, Heydari B. Relationship between different dimensions of prayer and spiritual health 
in hemodialysis patients. Iranian J Critical Care Nurs 2009; 2: 149-52. (Persian).

48. Askari P, Ruoshani Kh, Mehri Aderyani M. Relationship between religious reliefs and optimism with spiritual health in Ahvaz Islamic Azad university Students. J Yaftehaye nou dar Ravanshenasi 2010;27: 40. (Persian).

49. Holt CL, Kyles A, Wiehagen T, Casey C. Development of a spiritually based breast cancer educational booklet for AfricanAmerican women. Cancer Control 2003; 10: 37-44.
50. Quality of life in breast cancer patients: Study in the Omid cancer research center- UrmiaIranian Journal of Breast Diseases.43-35: (4) 5 ;2013

How to cite this Article: Madmoli M. A Systematic Review Study on the Changer Factors of the Quality of life in Cancer Patients. Int. Res. Med. Health Sci., 2019; 2(1):8-15.

Source of Support: Nil, Conflict of Interest: None declared. 